\title{
Deep Red Iridium(III) Complexes Based on Pyrene-substituted Quinoxaline Ligands for Solution-Processed Phosphorescent Organic Light-Emitting Diodes
}

\author{
Zhaoran Hao, ${ }^{\mathrm{b}}$ Kai Zhang, ${ }^{\mathrm{a}} \mathrm{Pu}$ Wang, ${ }^{\mathrm{b} *}$ Xumin Lu, ${ }^{\mathrm{b}}$ Zhiyun Lu, ${ }^{\mathrm{c} *}$ \\ Weiguo Zhu, a* Yu Liu ${ }^{\text {a,b* }}$ \\ a School of Materials Science and Engineering, Jiangsu Collaboration Innovation \\ Center of Photovoltaic Science and Engineering, Jiangsu Engineering Laboratory of \\ Light-Electricity-Heat Energy-Converting Materials and Applications, National \\ Experimental Demonstration Center for Materials Science and Engineering, \\ Changzhou University, Changzhou 213164, P. R. China. \\ ${ }^{b}$ College of Chemistry, Key Lab of Environment-Friendly Chemistry and Application \\ in Ministry of Education, Xiangtan University, Xiangtan 411105, China \\ c Key Laboratory of Green Chemistry and Technology of Ministry of Education, \\ College of Chemistry, and State Key Laboratory of Polymer Materials Engineering, \\ Sichuan University, Chengdu 610065, China \\ * Corresponding authors.
}

Email addresses:

(P.W.)947041300@qq.com,

(Z. L.) luzhiyun@scu.edu.cn,

(G. Z.) zhuwg18@126.com

(Y. L.)liuyu03b@126.com 


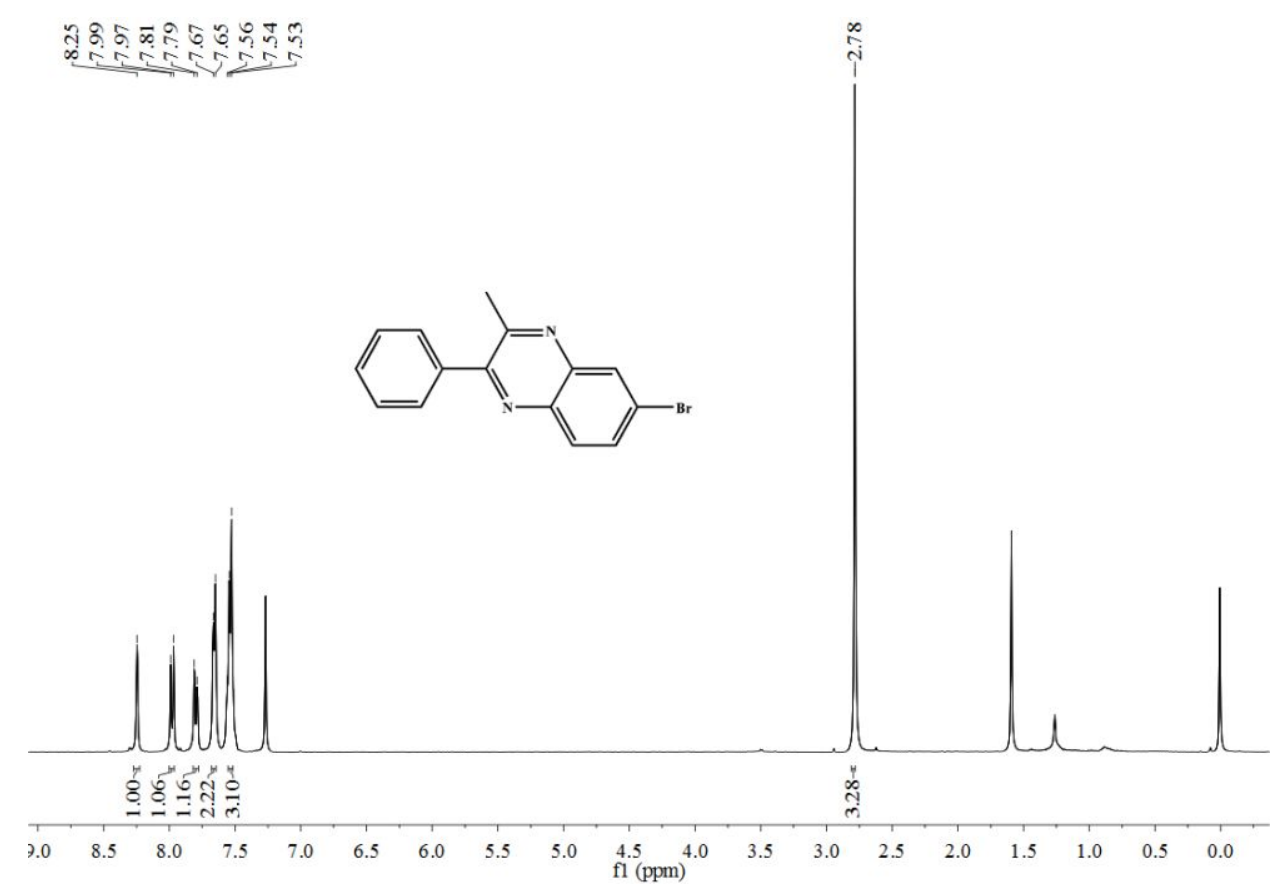

Figure S1 ${ }^{1} \mathrm{H}$ NMR spectrum of 6-bromo-3-methyl-2-phenylquinoxaline (M1).

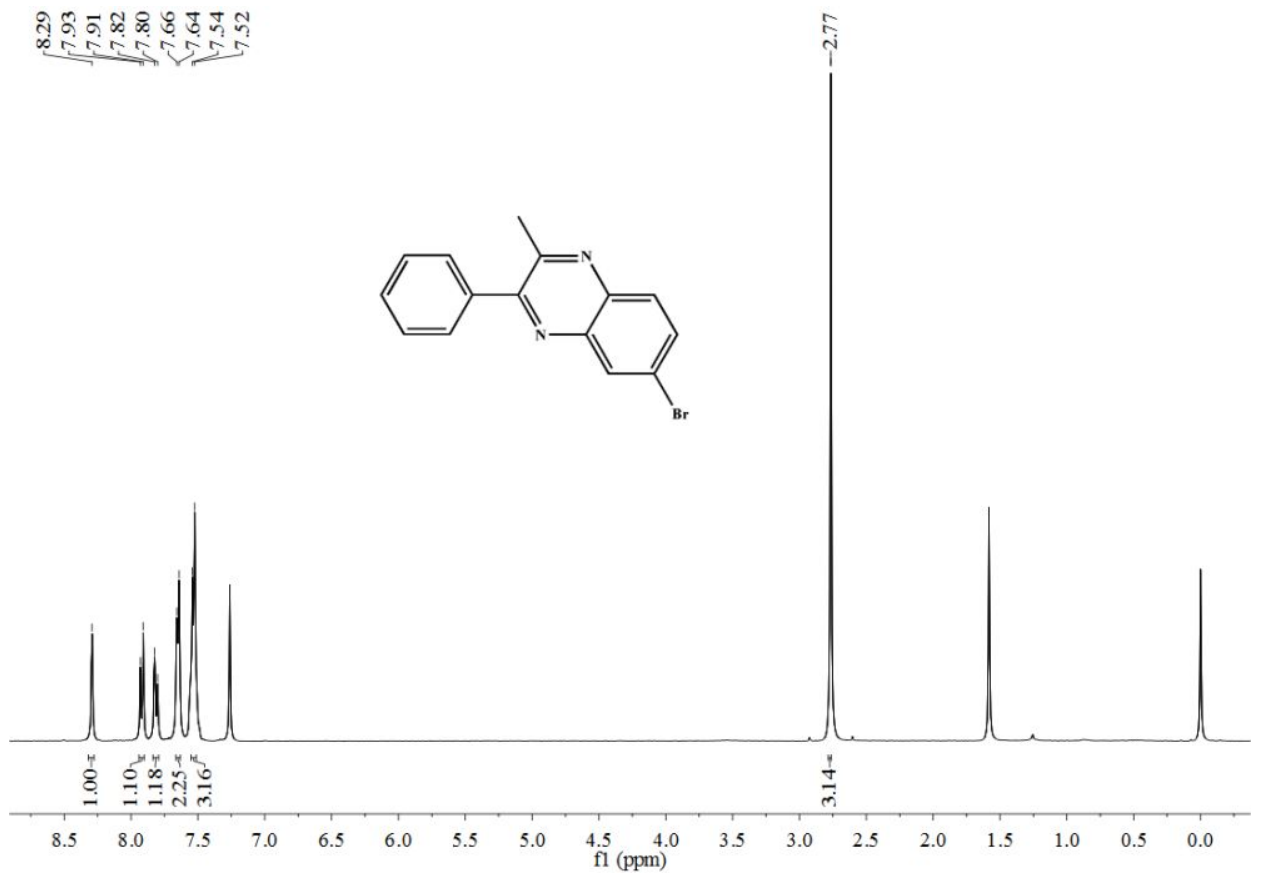

Figure S2 ${ }^{1} \mathrm{H}$ NMR spectrum of 6-bromo-2-methyl- 3-phenylquinoxaline (M2). 


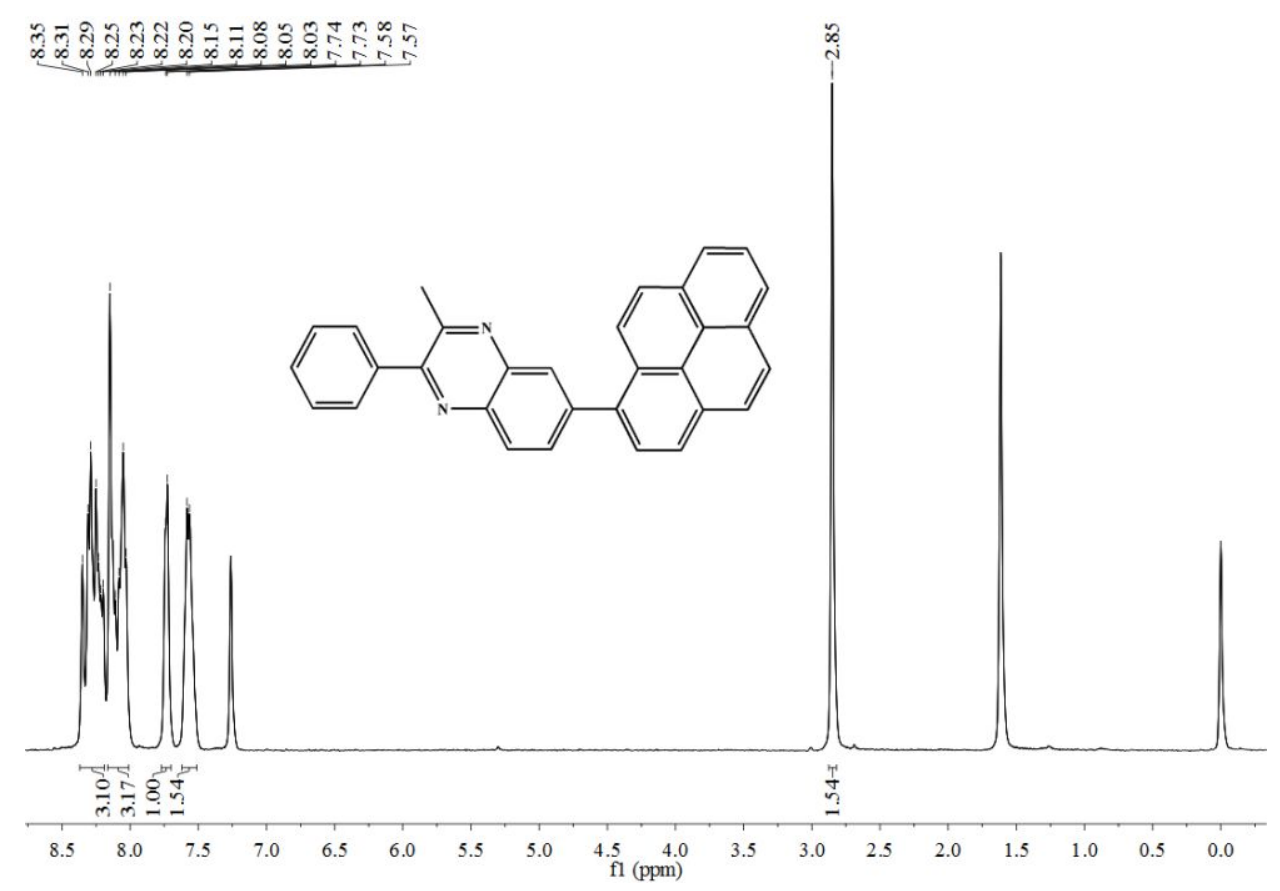

Figure S3 ${ }^{1} \mathrm{H}$ NMR spectrum of 3-methyl-2-phenyl-6-(pyren-1-yl)quinoxaline (c-PyMPQ).

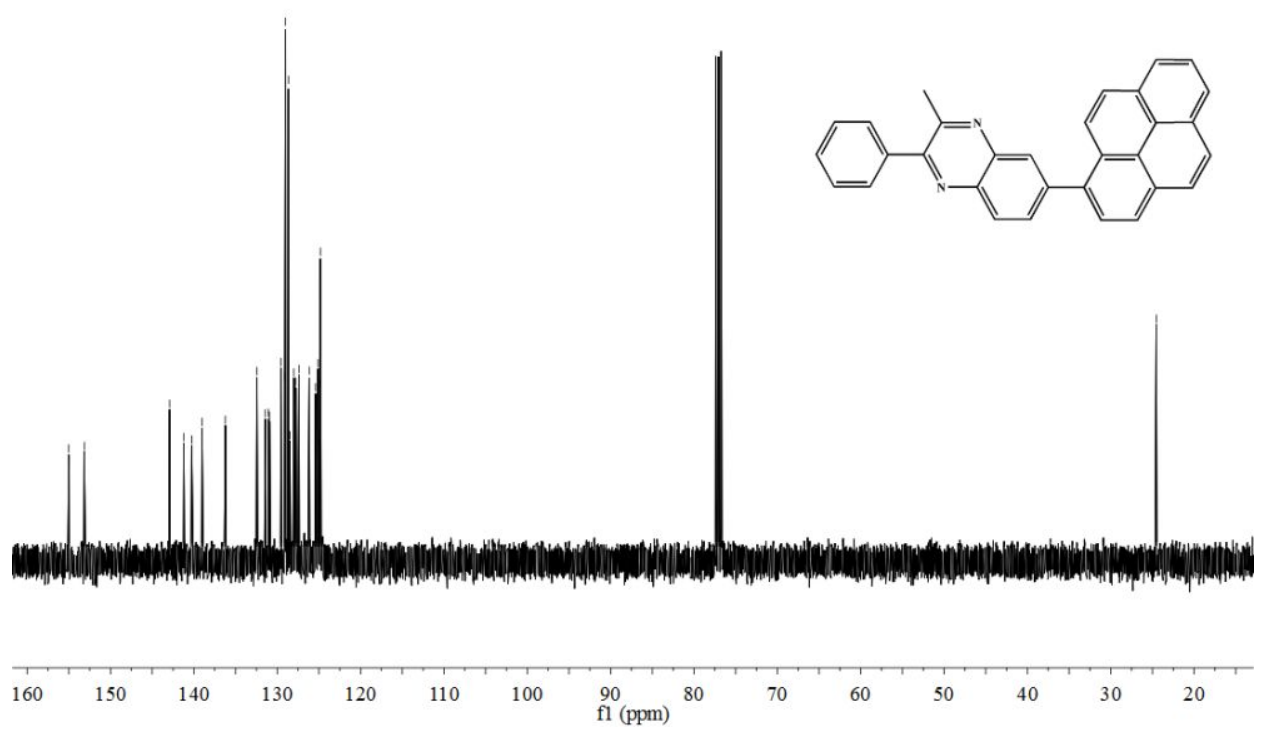

Figure S4 ${ }^{13} \mathrm{C}$ NMR spectrum of 3-methyl-2-phenyl-6-(pyren-1-yl)quinoxaline (c-PyMPQ). 


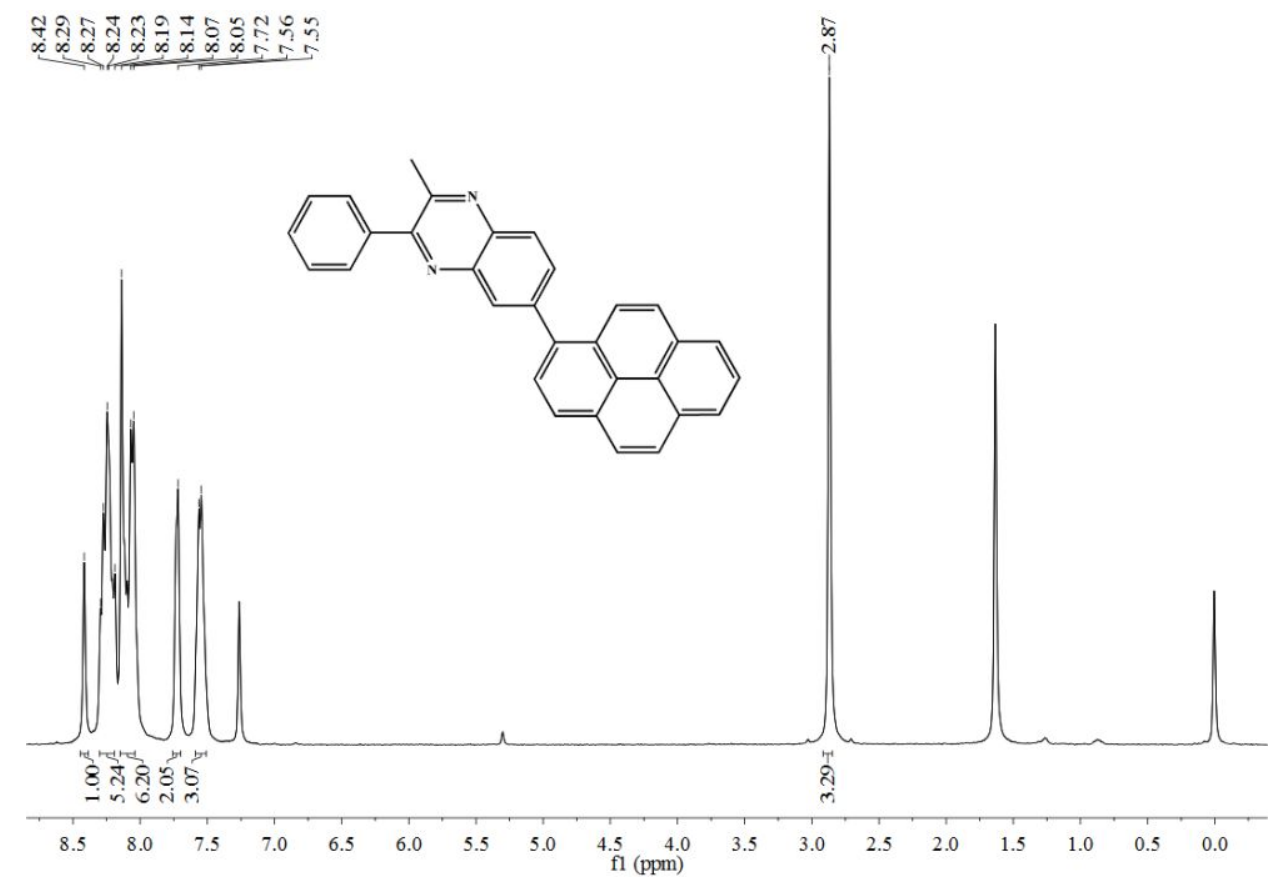

Figure S5 ${ }^{1} \mathrm{H}$ NMR spectrum of 2-methyl-3-phenyl-6-(pyren-1-yl)quinoxaline (t-PyMPQ).
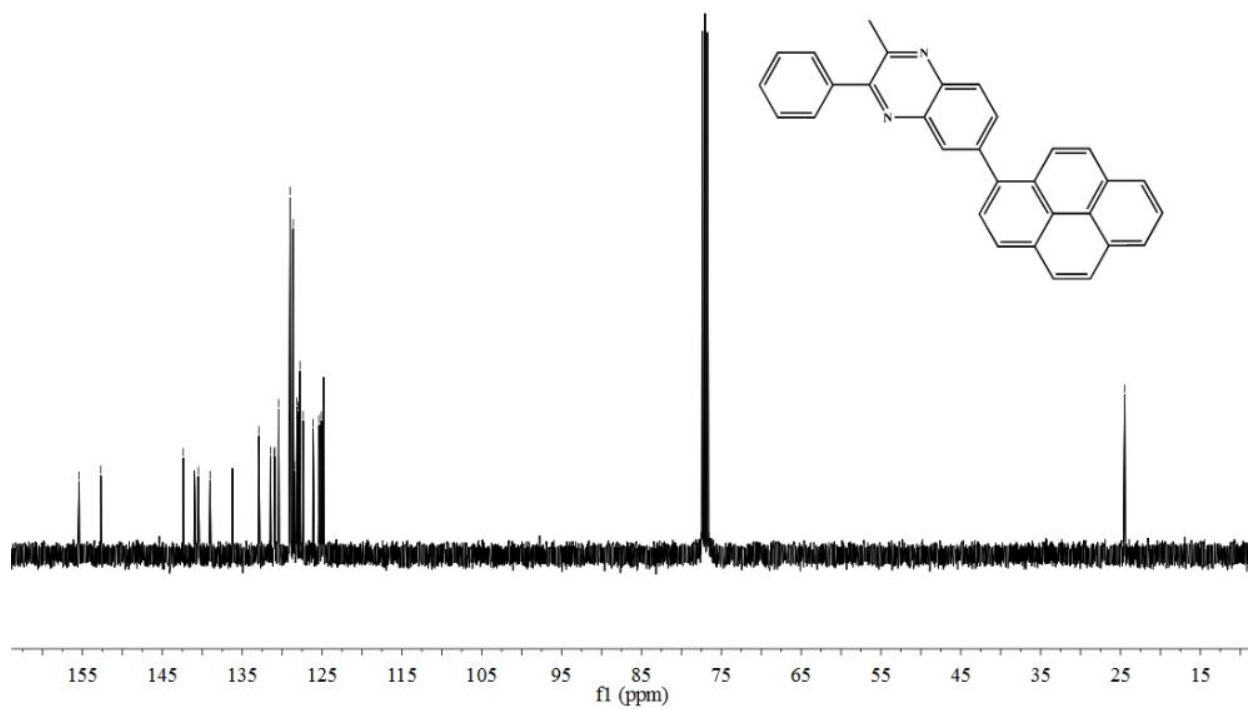

Figure S6 ${ }^{13} \mathrm{C}$ NMR spectrum of 2-methyl-3-phenyl-6-(pyren-1-yl)quinoxaline (t-PyMPQ). 


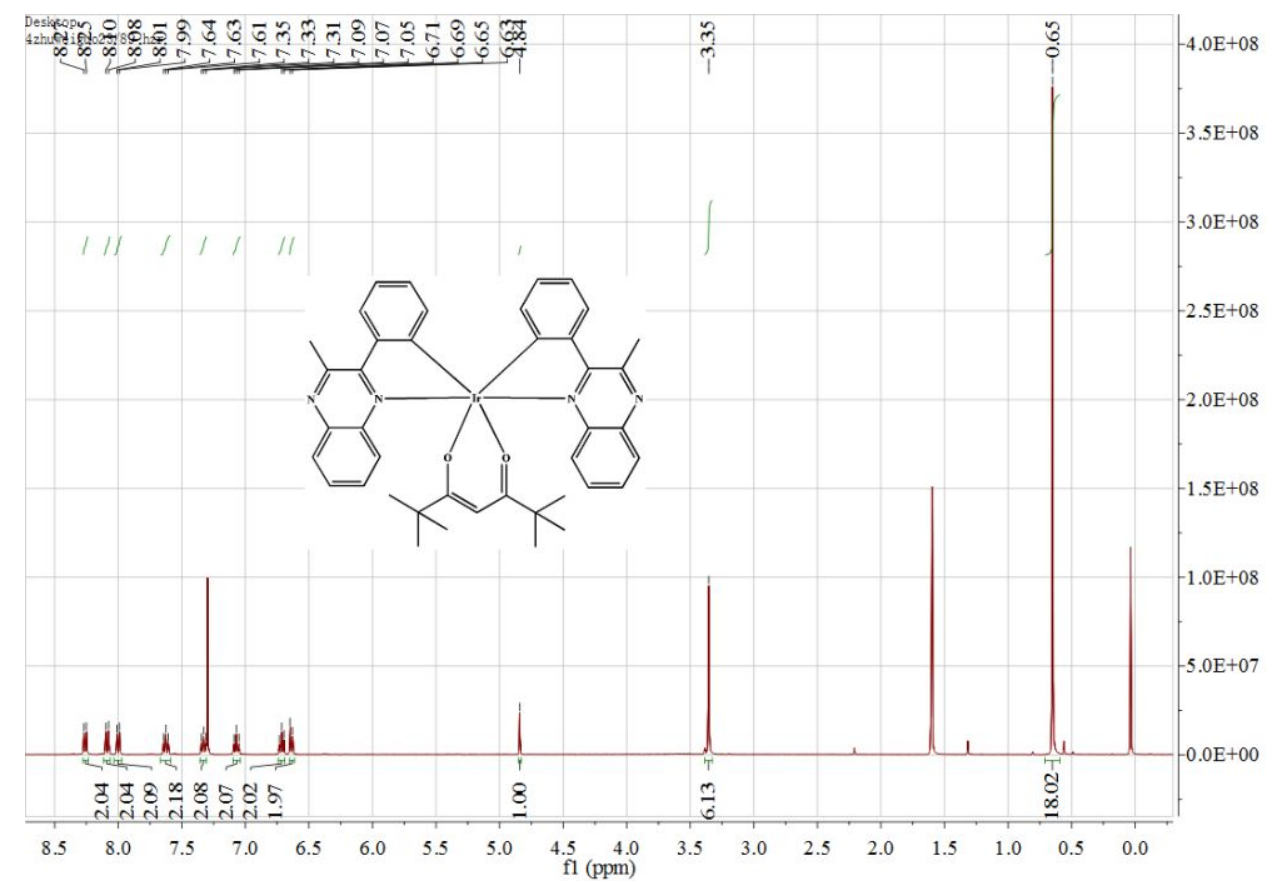

Figure S7 ${ }^{1} \mathrm{H}$ NMR spectrum of complex Ir-0.

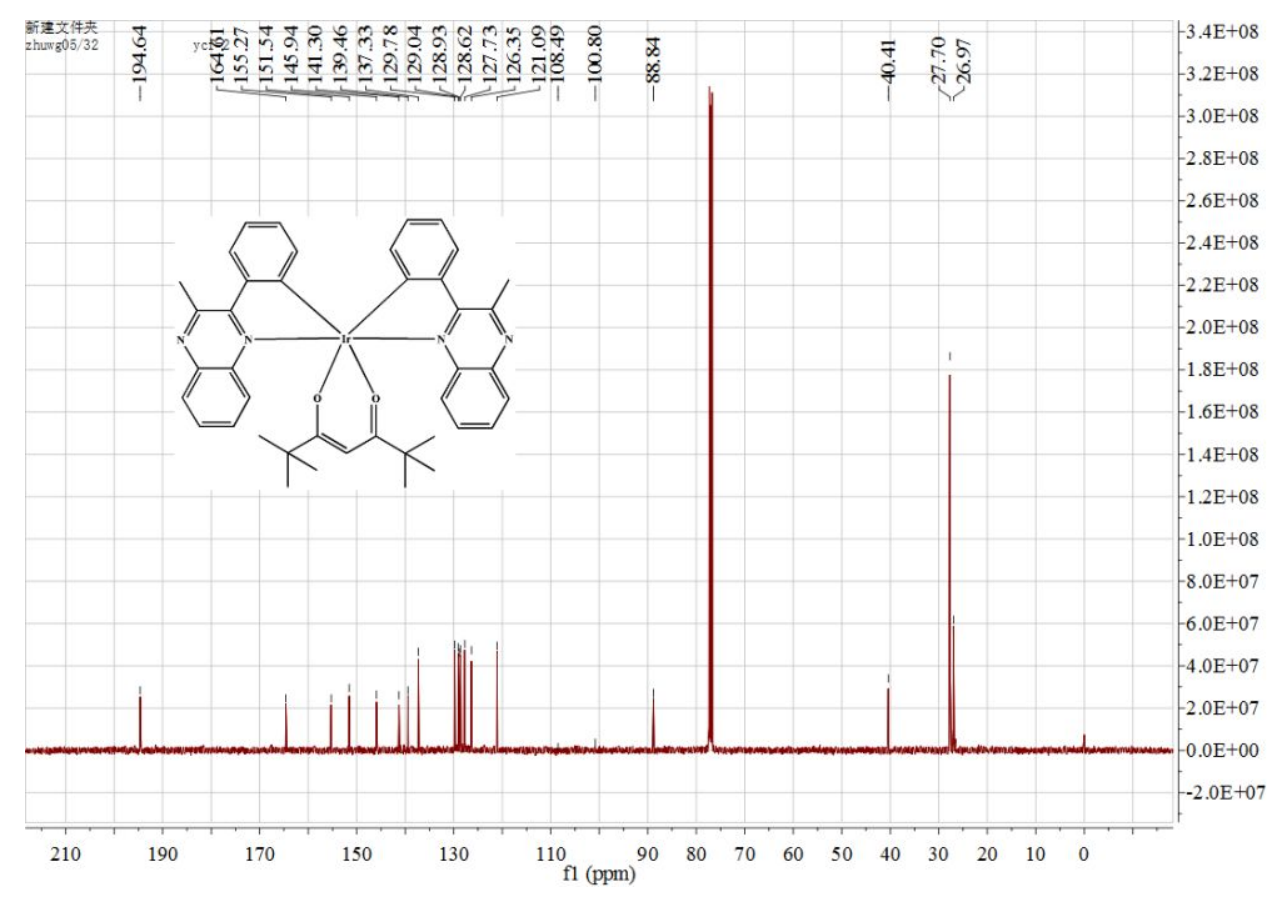

Figure S8 ${ }^{13} \mathrm{C}$ NMR spectrum of complex Ir-0. 


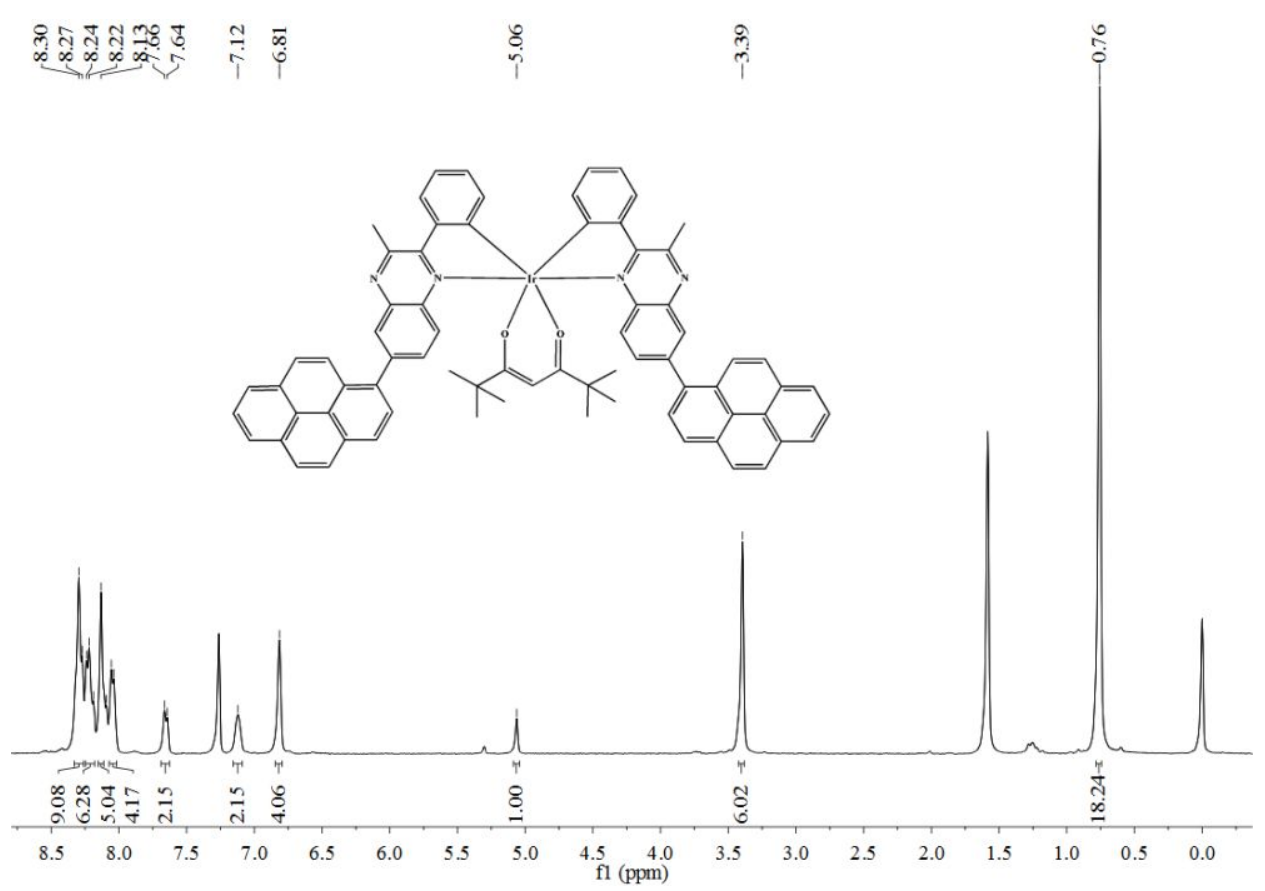

Figure S9 ${ }^{1} \mathrm{H}$ NMR spectrum of complex Ir-1.

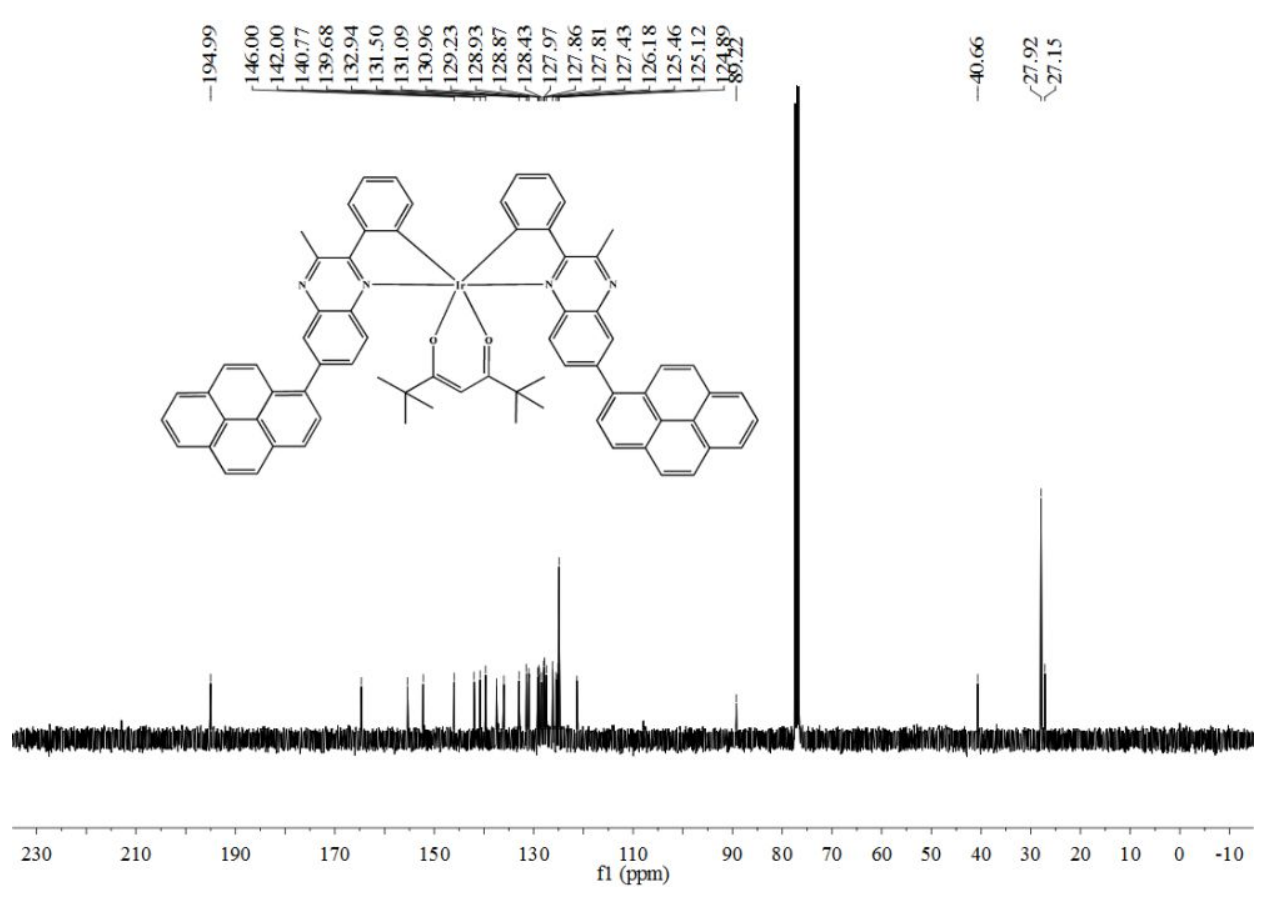

Figure S10 ${ }^{13} \mathrm{C}$ NMR spectrum of complex Ir-1. 


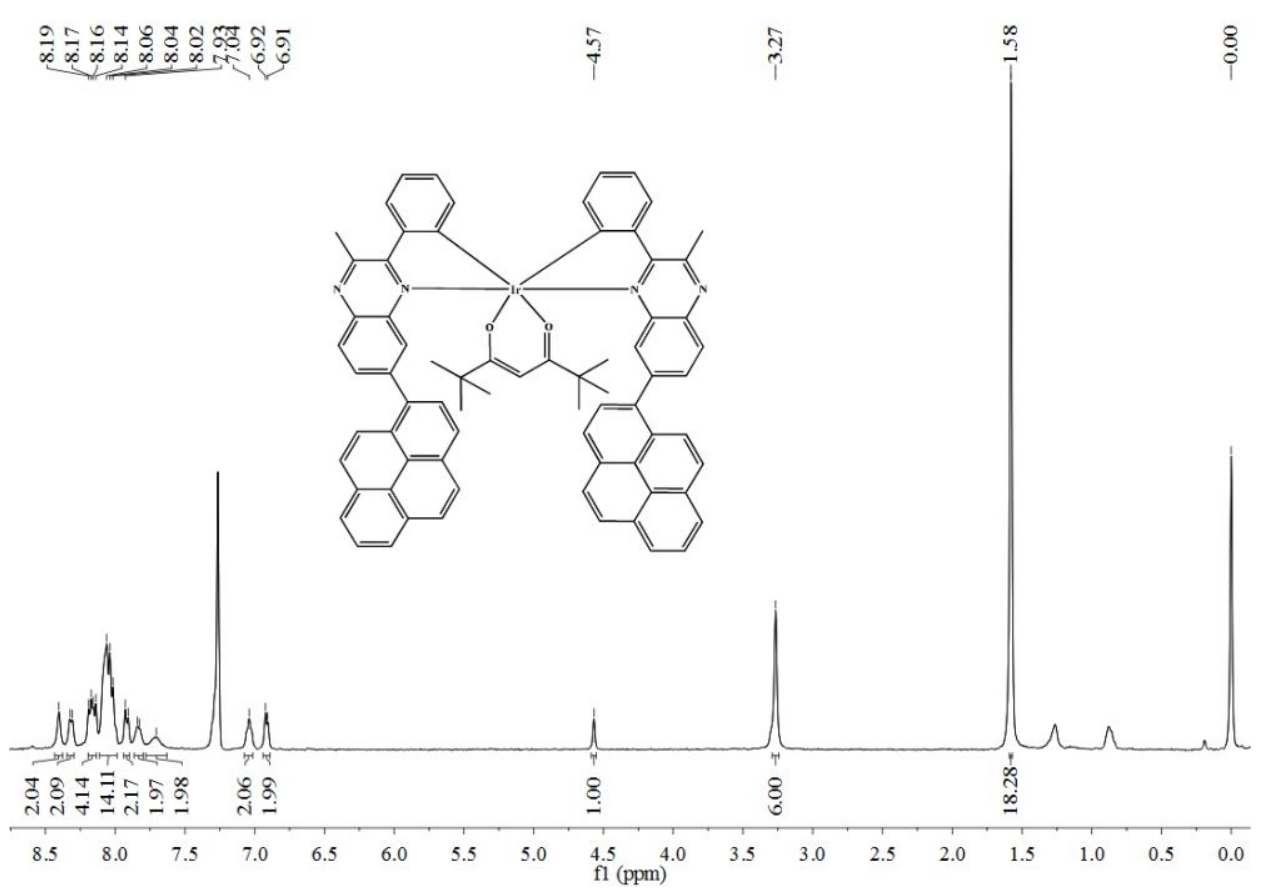

Figure S11 ${ }^{1} \mathrm{H}$ NMR spectrum of complex Ir-2.

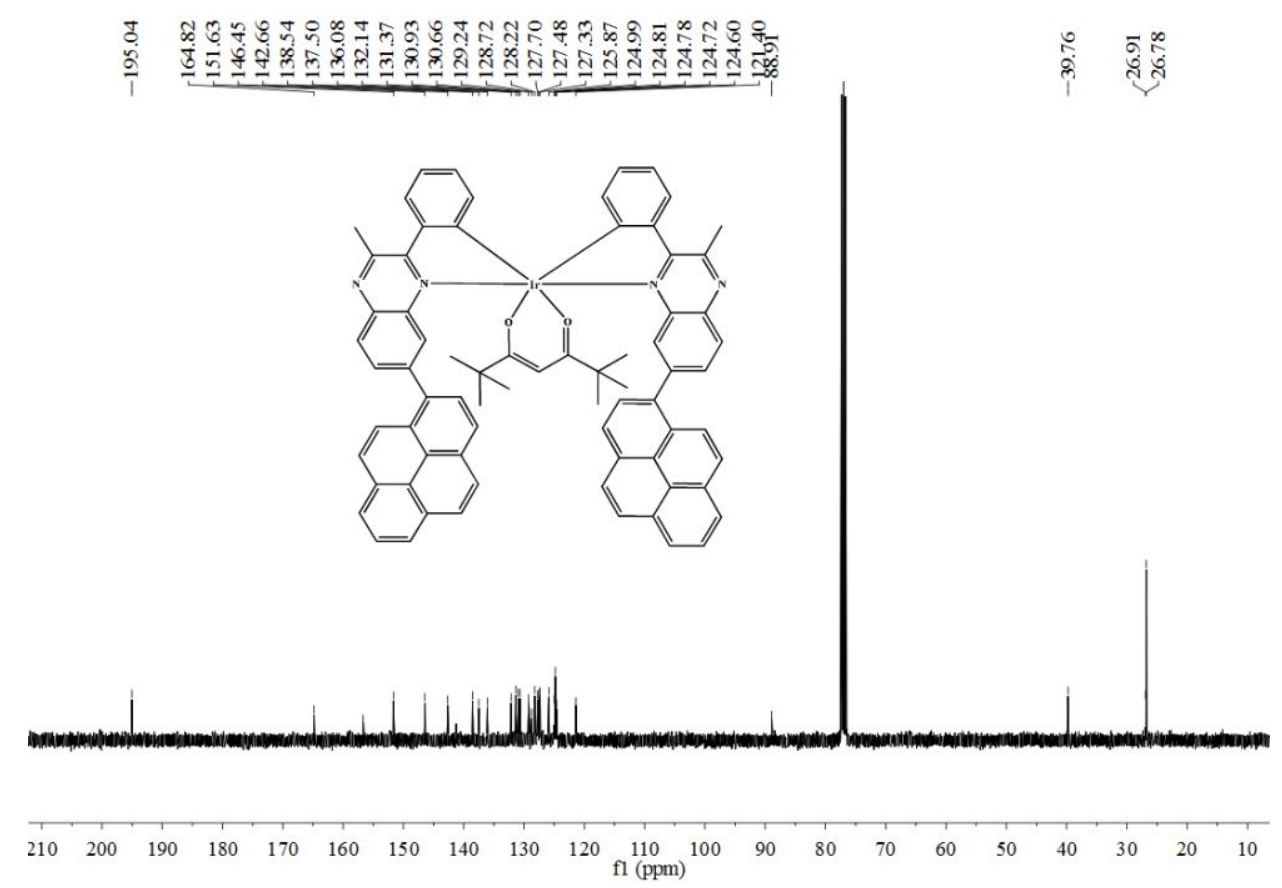

Figure S12 ${ }^{13} \mathrm{C}$ NMR spectrum of complex Ir-2. 

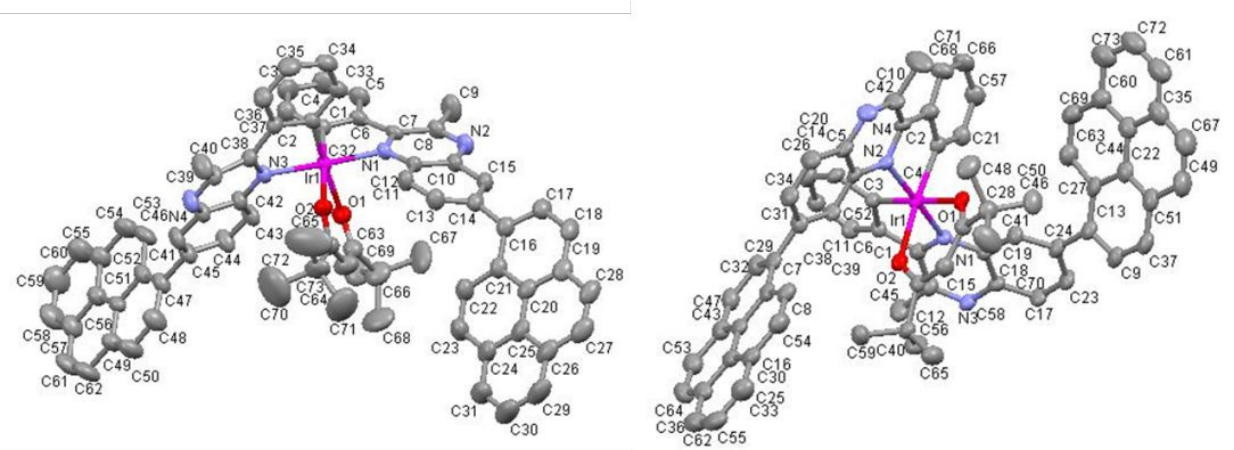

Figure S13. Single-Crystal structures of complexes, the thermal ellipsoids are drawn with $50 \%$ probability, and the hydrogen atoms and the solvent are omitted for clarity. Element (color): iridium (purple), oxygen (red), carbon (gray), nitrogen (blue). Ir-1 (left, bond lengths and angles at Ir ( $\mathrm{A}^{\circ}$, deg): Ir-N1, 2.048; Ir-N3, 2.052; Ir-C1, 1.978; Ir-C32, 1.976; Ir-O1, 2.166; Ir-O2, 2.168; N2-Ir-C4, 79.6; O1-Ir-O2, 85.1; N1-Ir-C3, 79.1) and Ir-2 (right, bond lengths and angles at $\operatorname{Ir}\left(\mathrm{A}^{\circ}, \mathrm{deg}\right)$ : Ir-N1, 2.068; Ir-N2, 2.068; Ir-C3, 1.961; Ir-C4, 1.986; Ir-O1, 2.151; Ir-O2, 2.139; N1-Ir-C1, 79.1; O1-Ir-O2, 86.6; N3-Ir-C32, 80.1).

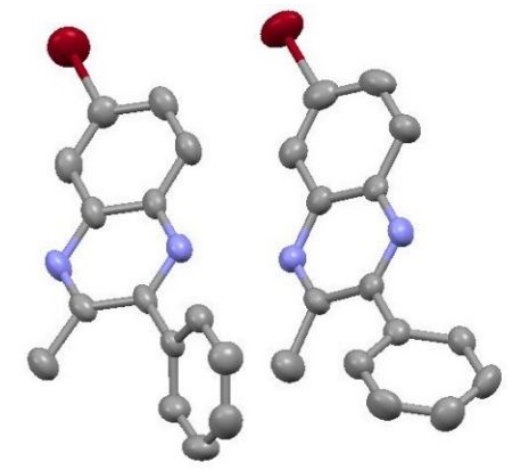

Figure S14. Single-Crystal structures of 6-bromo-3-methyl-2-phenylquinoxaline racemic mixtures, the thermal ellipsoids are drawn with $50 \%$ probability; the hydrogen atoms and the solvent are omitted for clarity. Element (color): bromine (red), carbon (gray), nitrogen (blue).

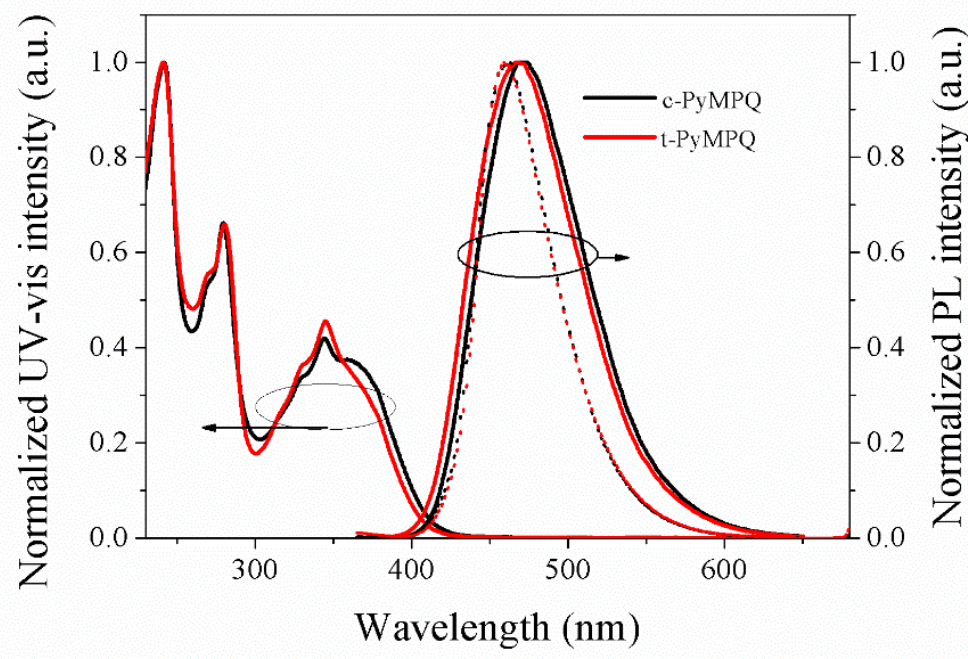

Figure S15 UV-Vis absorption and emission spectrum of free ligands c-PyMPQ and t-PyMPQ in dilute 2-MeTHF at rt (solid line) and $77 \mathrm{~K}$ (dot line). 


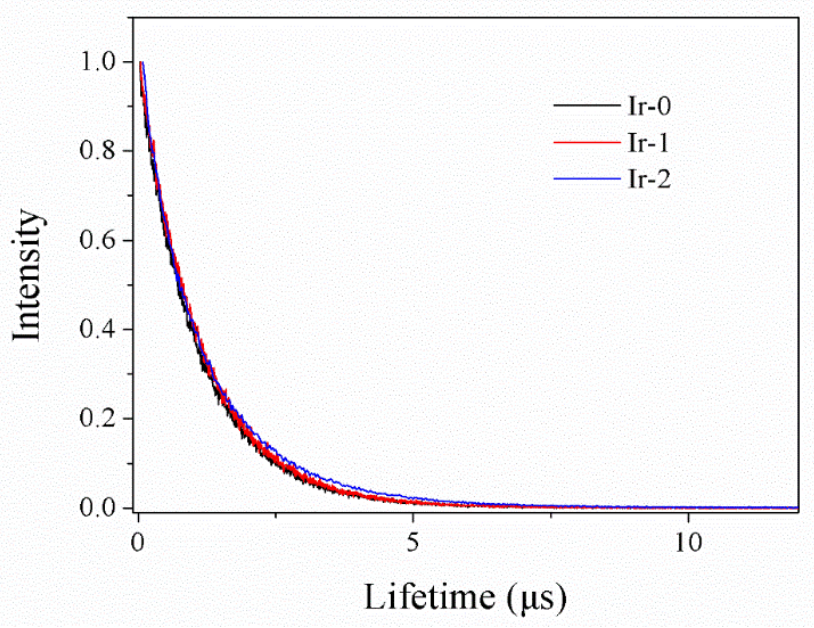

Figure S16 Emission decay curves of complexes in 2-MeTHF at RT.

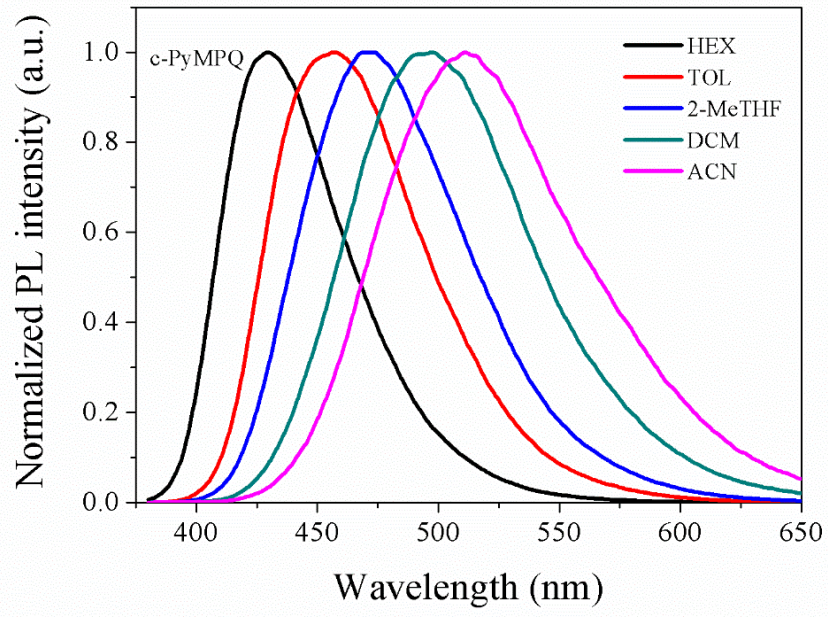

Figure S17 Emission spectrum of free ligands of c-PyMPQ in different solvent.

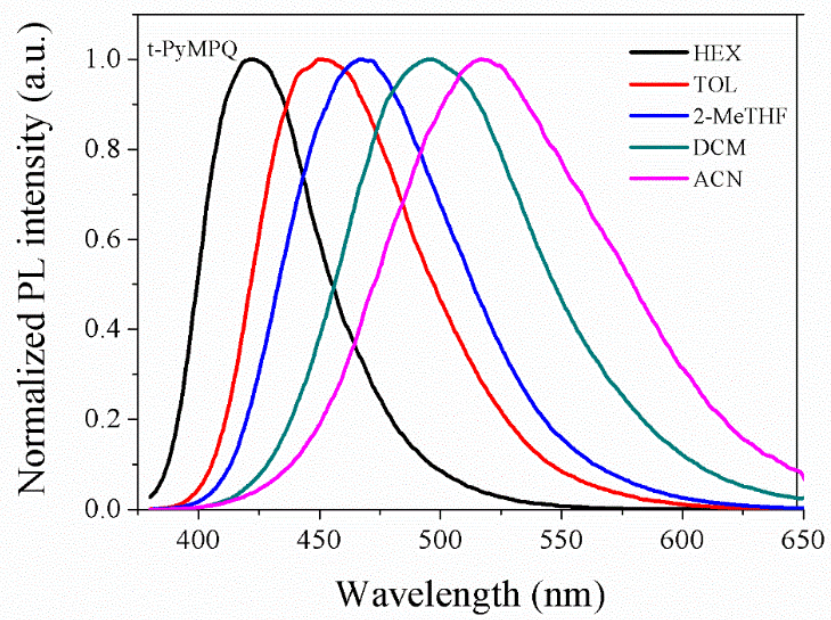

Figure S18 Emission spectrum of free ligands of t-PyMPQ in different solvent. 


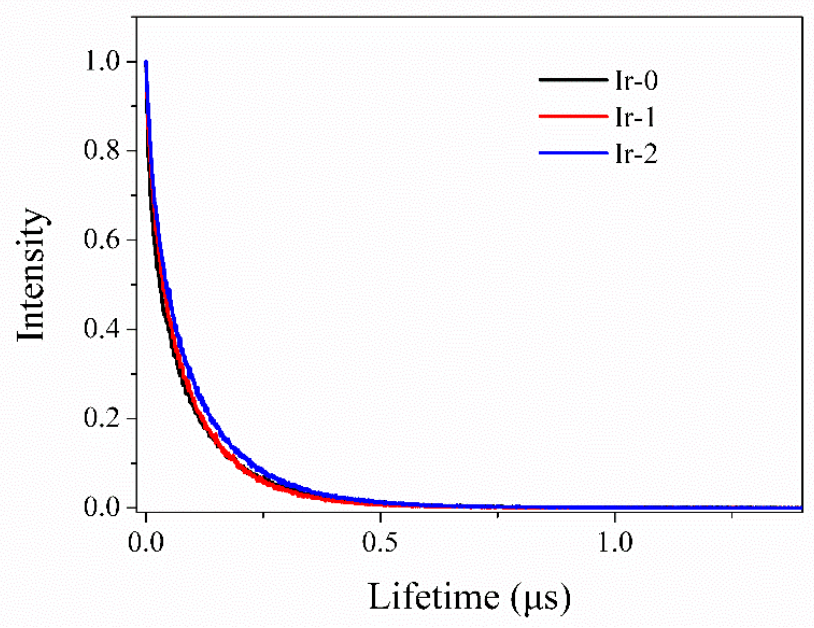

Figure S19 Emission decay curves of complexes in thin films.

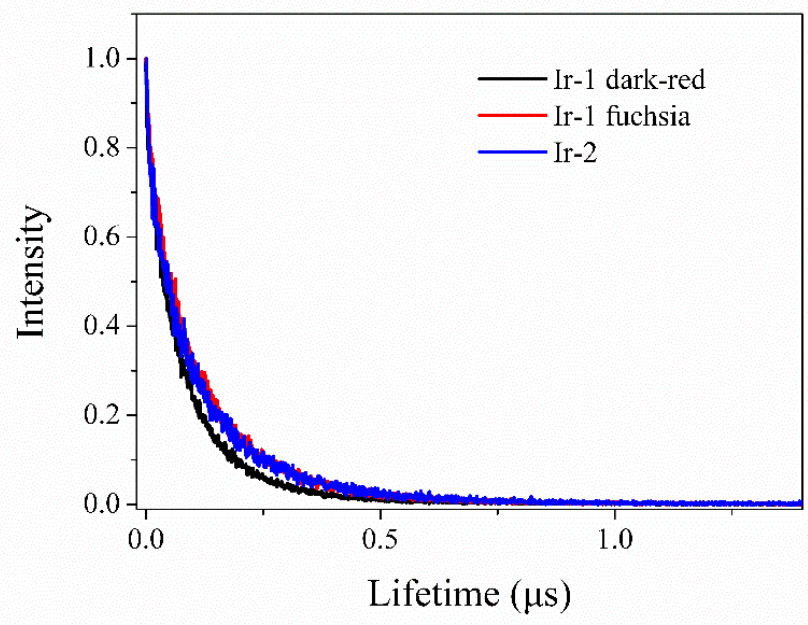

Figure S20 Emission decay curves of complexes in crystal powders.

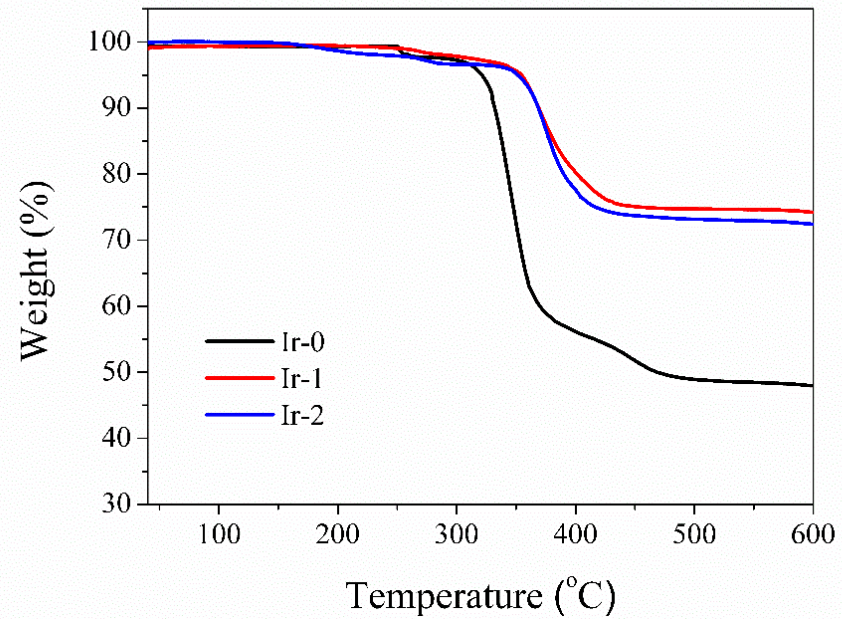

Figure S21 TGA curves of the complexes. 

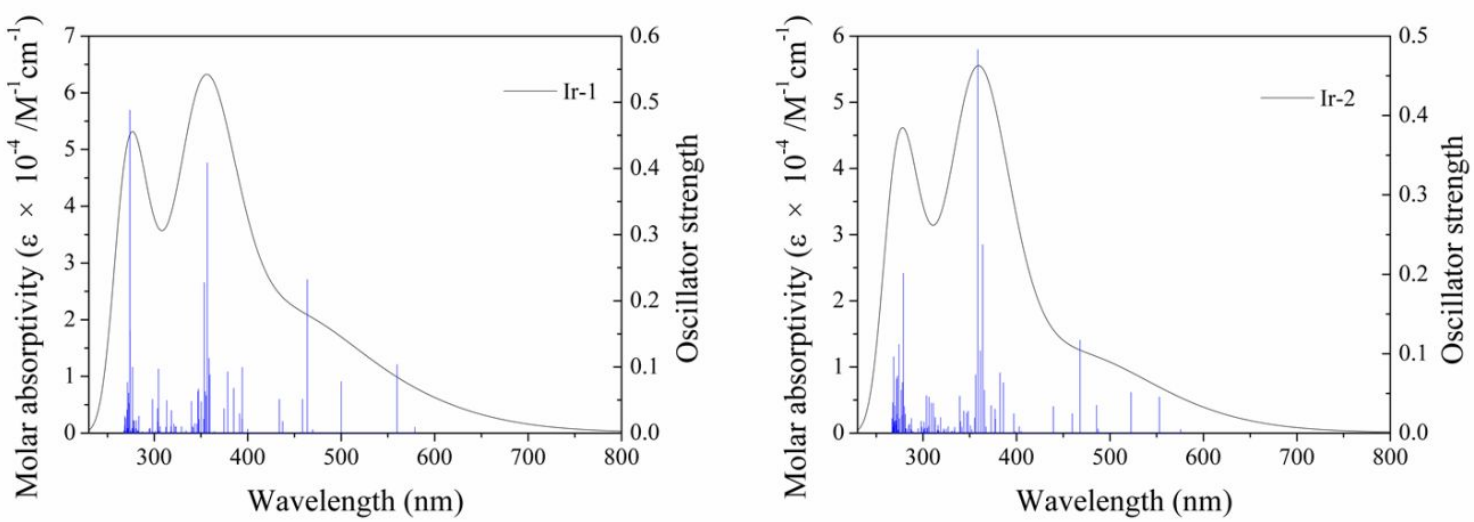

Figure S22 Calculated absorption spectra of Ir-1 and Ir-2, The oscillator strengths (vertical blue lines) are of the 50 lowest-energy calculated singlet-singlet transitions.

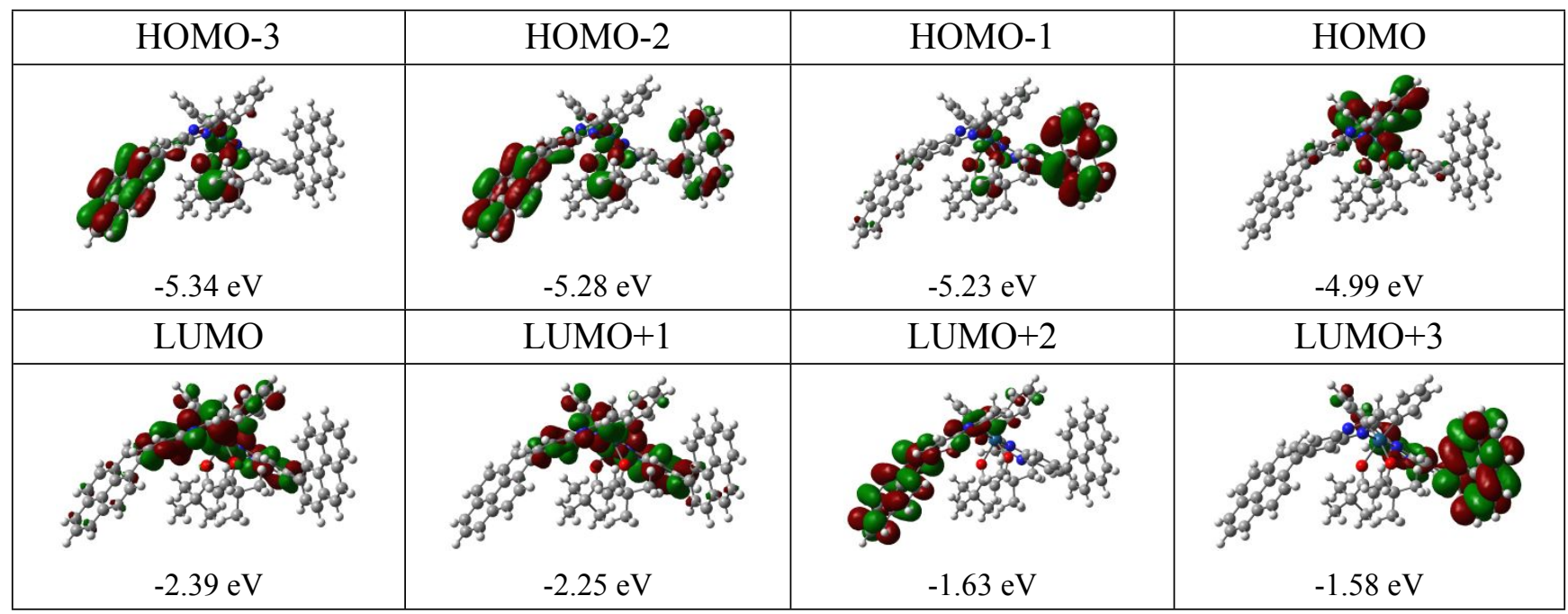

Figure S23 Molecular orbital energy diagrams of Ir-2 obtained at the UB3LYP theory level based on its optimized triplet state geometry.

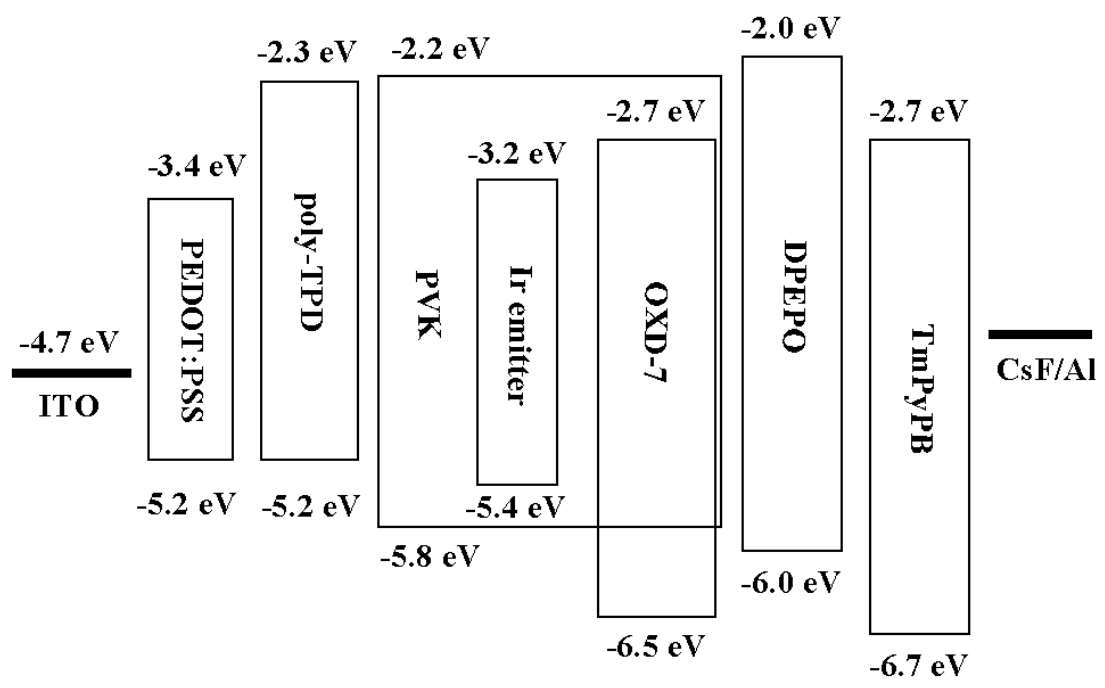

Figure S24 Devices structure and energy level diagram of complexes-doped devices. 

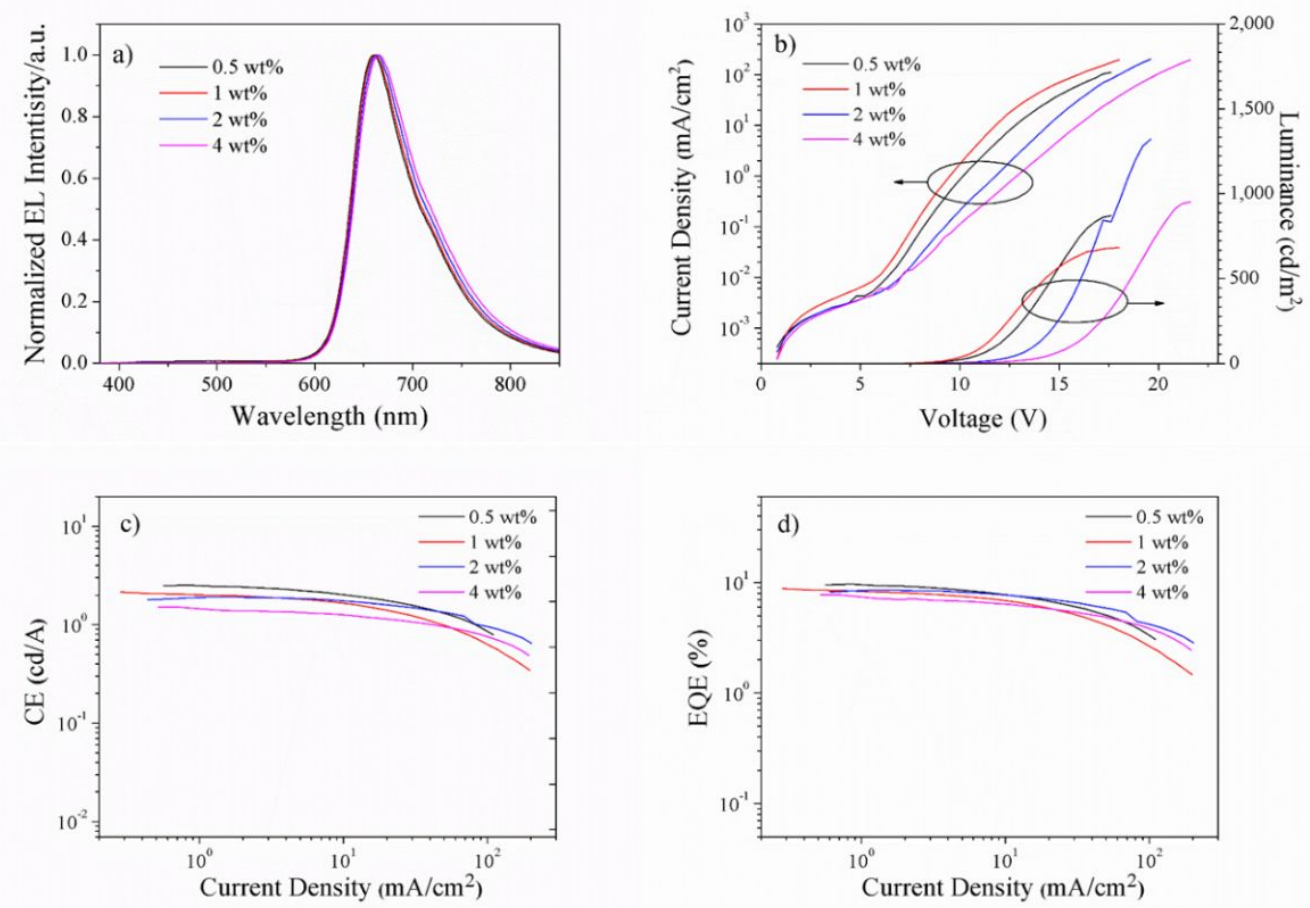

Figure S25 (a) EL spectra, (b) $J-V-R$ characteristics, (c) current density- $C E$, (d) current density-EQE curves of devices Ir-0 at different doping concentrations.
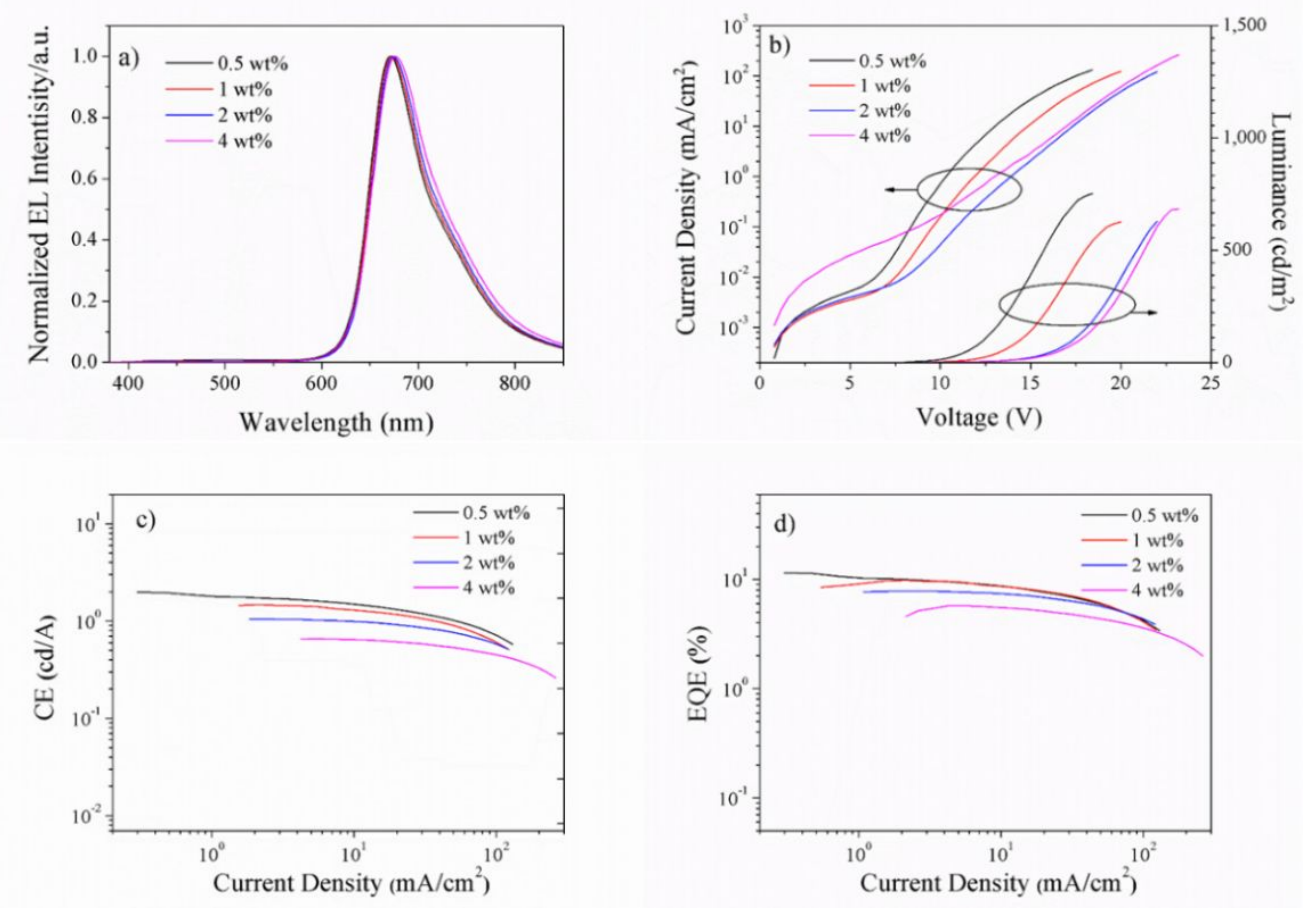

Figure S26 (a) (a) EL spectra, (b) $J-V-R$ characteristics, (c) current density- $C E$, (d) current density-EQE curves of devices Ir-1 at different doping concentrations. 

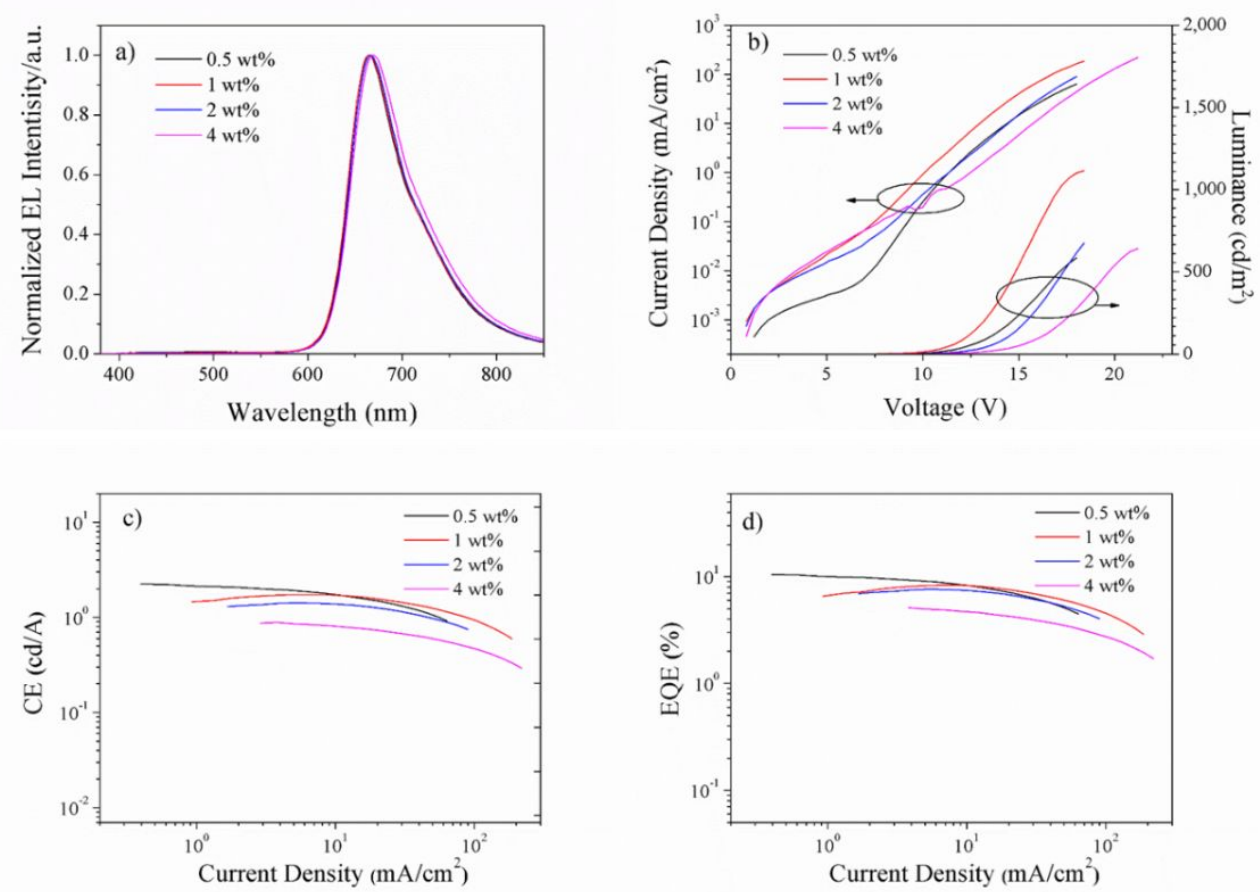

Figure S27 (a) EL spectra, (b) $J-V-R$ characteristics, (c) current density- $C E$, (d) current density-EQE curves of devices Ir-2 at different doping concentrations. 